\title{
Caracterización del concepto de contabilidad simplificada en España y Colombia
}

\author{
Diego Fernando Católico Segura* \\ Fabio Enrique Gómez Meneses ${ }^{\star *}$
}

\begin{abstract}
Católico Segura, D.F. y Gómez Meneses, F.E. (2011). Caracterización del concepto de contabilidad simplificada en España y Colombia. Revista Activos, 16 y 17, 71-98.
\end{abstract}

JEL: M41

Recibido: 4 de abril de 2011 Aprobado: 12 de mayo de 2011

\section{Resumen}

En el presente documento se analizará el concepto de contabilidad simplificada en España y Colombia, identificado su génesis, características y el tipo de organizaciones para las cuales puede resultar útil; organizaciones que corresponden principalmente a las micro, pequeñas y medianas empresas. Todo lo anterior, en el

* Contador Público, Universidad Nacional de Colombia; Especialista en Finanzas, Universidad Santo Tomás; Máster en Contabilidad y Finanzas y Doctorando en Contabilidad y Finanzas de la Universidad de Zaragoza, España. Docente de tiempo completo de la Facultad de Ciencias Administrativas y Contables de la Universidad de La Salle.

** Contador Público y Especialista en Revisoría Fiscal y Auditoría Externa, Universidad Autónoma de Bucaramanga; Máster en Administración Electrónica de Empresas, Máster en Contabilidad y Finanzas y Doctor en Contabilidad y Finanzas de la Universidad de Zaragoza, España. Profesor de tiempo completo de la Facultad de Contaduría Pública de la Universidad Santo Tomás, Bogotá. 
contexto del proceso actual de estandarización contable internacional por el que atraviesa nuestro país con ocasión de lo estipulado en la Ley 1314 de 2009. En el artículo se tienen en cuenta las ventajas y desventajas que dicho proceso supone y las particularidades de las organizaciones en las cuales se pretenda utilizar dicho sistema.

\title{
Palabras clave
}

Contabilidad simplificada, modelos contables, grupos de interés, agentes económicos, MiPymes.

Católico Segura, D.F. y Gómez Meneses, F.E. (2011). Caracterization of the Concept of Simplified Accountancy in Spain and Colombia. Activos Review, 16 and 17, 71-98.

\begin{abstract}
This paper analyzes the concept of simplified accounting in Colombia and Spain, identifying its origin, features and kind of organizations that could find it helpful; organizations that are mainly micro, small and medium size enterprises. All of this from the perspective of the current international accounting standardization process that is having place in our country according to the Law 1314 of 2009. In the document are taken into account the advantages and disadvantages of the process and the special features of the organizations that will use this system.
\end{abstract}

\section{Keywords}

Simplified accountancy, accounting models, interest groups, economical agents, SME.

Católico Segura, D.F. y Gómez Meneses, F.E. (2011). Caractérisation du concept de comptabilité simplifiée en Espagne et en Colombie. Revue Activos, 16 et 17, 71-98. 


\section{Résumé}

Dans le présent document, le concept de comptabilité simplifiée en Espagne et en Colombie sera analysé, son origine, ses caractéristiques et le type d'organisations pour lesquelles il peut s'avérer utile seront identifiés, organisations qui correspondent principalement aux micro, petites et moyennes entreprises, ceci dans le contexte du processus actuel de standardisation comptable internationale que traverse notre pays selon ce que stipule la loi 1314 de 2009, en prenant en compte les avantages et les inconvénients que ledit processus suppose et les particularités des organisations dans lesquelles on prétend utiliser ce système.

\section{Mots-clés}

Comptabilité simplifiée, modèles comptables, groupes d’intérêt, agents économiques, MIPMES.

\section{Introducción}

Colombia se encuentra en una etapa de ajuste de sus normas de contabilidad respecto a estándares internacionales, a partir de lo señalado en la Ley 1314 de 2009. Sin embargo, los ajustes y los caminos que se puedan plantear para atender lo establecido en la ley tienen importantes ventajas y desventajas, dadas las intencionalidades que tiene la norma, pero, a su vez, las características administrativas, económicas y jurídicas de las organizaciones.

La normatividad contable vigente, sustentada principalmente en el Decreto 2649 de 1993, Decreto 2650 de 1993 y en los lineamientos expuestos en los últimos años por las distintas Superintendencias y por la Dirección de Impuestos y Aduanas Nacionales (DIAN), ha sido objeto de críticas a causa de su atraso para responder a las condiciones actuales de los negocios de las organizaciones, así como por su disparidad y su dispersión entre los reguladores (World Bank, 2003). Para esto, se propone una serie de ajustes que atiendan a estos señalamientos y a las tendencias internacionales de convergencia, para propiciar una comparabilidad de la información contable. 
No obstante, al atender estas circunstancias a través de los ajustes que resulten necesarios frente a las normas contables, es importante reconocer las características del entorno socio-económico colombiano, así como las condiciones propias de las organizaciones, las cuales inciden en el tipo de información que desde la contabilidad se debe preparar y presentar para el uso por parte de los distintos usuarios o grupos de interés de las empresas.

Es claro que en nuestro país la gran mayoría de empresas corresponden a MiPymes. En este sentido, es importante estudiar el tipo de contabilidad planteada para esta población de compañías colombianas, como efectivamente es abordado en el artículo segundo de la Ley 1314 de 2009, en el que textualmente se plantea:

En atención al volumen de sus activos, de sus ingresos, al número de sus empleados, a su forma de organización jurídica o de sus circunstancias socioeconómicas, el Gobierno autorizará de manera general que ciertos obligados lleven contabilidad simplificada, emitan estados financieros y revelaciones abreviados o que estos sean objeto de aseguramiento de información de nivel moderado.

En desarrollo de programas de formalización empresarial o por razones de política de desarrollo empresarial, el Gobierno establecerá normas de contabilidad y de información financiera para las microempresas, sean personas jurídicas o naturales, que cumplan los requisitos establecidos en los numerales del artículo 499 del Estatuto Tributario.

De acuerdo a lo planteado en la norma citada, para aquellas empresas y según su actividad económica y su tamaño (micro, pequeña o mediana empresa), el Gobierno Nacional planteará un esquema de contabilidad simplificada para efectos de la información que deben reportar.

Teniendo en cuenta lo anterior, podemos plantear en el desarrollo de este trabajo la siguiente pregunta de investigación: ¿cuál es el concepto y las características de la contabilidad simplificada en algunos de los países que manejan este concepto? 
A partir de este interrogante se busca identificar las características de la contabilidad simplificada, teniendo como referente los desarrollos que sobre dicho tema se han planteado en países como Colombia y España ${ }^{1}$. De allí que la indagación inicial sobre normas y estudios desarrollados al respecto serán la fuente de información principal para identificar las características propias de dicho concepto.

\section{Marco teórico}

$\mathrm{Al}$ entender la contabilidad como un sistema de información sobre los eventos económicos que realizan las organizaciones en el desarrollo de sus objetos sociales, ésta sirve como instrumento para la toma de decisiones de los diferentes grupos de interés frente a los recursos que las empresas controlan o poseen. Es importante señalar que los lineamientos que se establecen para definir cómo se prepara y presenta la información que surge de este sistema dependen de las características del entorno, de las necesidades de información de los distintos usuarios que tienen interés en las organizaciones y de las condiciones internas de estas últimas.

Visto lo anterior desde un enfoque lógico-deductivo y teleológico (Cañibano, 1996), los lineamientos conceptuales y normativos que definen la forma como se hace la contabilidad financiera en los entes económicos hacen referencia a: la identificación de las necesidades de información de los usuarios internos y externos de las organizaciones; los objetivos que se proponen para la información contable a partir de las necesidades de información reconocidas; las cualidades que son atributos esenciales para cumplir los objetivos establecidos y, por último, los elementos y los criterios de representación, medición, valoración y revelación de la información.

Esta forma de establecer los lineamientos conceptuales para realizar la contabilidad en las organizaciones reconoce los desarrollos teóricos existentes, con el fin de determinar las prácticas de contabilidad adecuadas que se pueden plantear desde la regulación. Al respecto, Hendriksen plantea:

1 Se ha seleccionado a Colombia y España dado que son países que se caracterizan por pertenecer a un sistema contable de tipo continental europeo. Este sistema generalmente se identifica por factores tales como: la financiación de las empresas a través de recursos provistos por el sector financiero, la influencia de lo fiscal en lo contable y la presencia de un número importante de pequeñas y medianas empresas. 
Una estructura teórica lógicamente deducida para el desarrollo de prácticas de contabilidad apropiadas debe incluir las siguientes consideraciones:

1. Un acuerdo sobre postulados relacionados con el carácter de la entidad contable y su medio.

2. Una evaluación de las necesidades del usuario y sus constreñimientos en cuanto a su capacidad de comprender, interpretar y analizar la información que se le presenta.

3. La selección de lo que debe informarse. Esto debe incluir una selección de los objetivos y actividades de la entidad o su ambiente y los atributos específicos que son pertinentes a los objetivos de la contabilidad.

4. Una evaluación de los posibles procesos de medición y descripción para comunicar información sobre la empresa y su medio.

5. Una evaluación de los constreñimientos referentes a la medición y descripción de la entidad y su medio.

6. El desarrollo de principios o proposiciones generales que puedan usarse como directrices en la formulación de procedimientos y reglas.

7. La formulación de una estructura y un modelo para la recopilación y elaboración de datos y para resumir y dar cuenta de la información pertinente (1981: 109).

Según lo planteado, se debe tener en cuenta que la formulación de los conceptos antes expuestos demanda, entre otros aspectos, el reconocimiento de las necesidades de los usuarios de la información y los objetivos que dicha información cumple.

\section{Identificación de los grupos de interés en las organizaciones}

Las necesidades de los usuarios y los objetivos de la información se pueden estudiar desde la teoría contractual expuesta por Sunder (2005), en donde se entiende a las organizaciones como un conjunto de contratos entre individuos o grupos de individuos, con diversas necesidades de información. Desde esta teoría, la contabilidad ayuda a poner en práctica y a hacer cumplir los contratos que suscribe y constituye la empresa. Para ello, y según el autor, la contabilidad desempeña cinco funciones para posibilitar el funcionamiento de una organización, en función de buscar las condiciones deseadas de información para la ejecución de los contratos: 
1. Mide las contribuciones de cada agente en el fondo común de recursos de la empresa.

2. Determina y liquida el derecho contractual de cada agente.

3. Informa a los agentes apropiados hasta qué punto los otros agentes han cumplido sus obligaciones contractuales y han recibido sus derechos.

4. Ayuda a mantener un mercado líquido para las participaciones contractuales y para los factores de producción ofrecidos por sus participantes, de tal manera que la rescisión o terminación del contrato de un agente no amenace la existencia de la empresa.

5. Como los contratos de varios agentes son periódicamente renegociados, provee una base de conocimiento común de información verificada a todos los participantes, para facilitar la negociación y la formalización de los contratos.

En este sentido, la contabilidad, en tanto sistema de información, contribuye a la formalización, seguimiento y evaluación de los contratos que determinan las relaciones entre las organizaciones y los distintos agentes.

Tradicionalmente, las relaciones que se reconocen, como lo expone Toca (2007), son las establecidas con los propietarios, los proveedores, los acreedores, los empleados y los administradores. En el caso de los propietarios y accionistas, la relación contractual se sustenta dados los recursos que estos invierten en las organizaciones, esperando una retribución a través del pago de unos dividendos, del reconocimiento de unas ganancias de capital y de la maximización de su riqueza.

Los proveedores suministran recursos, conocimiento y/o habilidades con el fin de tener oportunidades para lograr beneficios razonables. Los acreedores, al proporcionar recursos financieros, esperan en el futuro pagos periódicos oportunos, que incluyen una remuneración de intereses y comisiones por el servicio prestado. Los empleados son las personas que entregan su mano de obra y contribuyen con sus conocimientos en la gestión de procesos, recibiendo a cambio una serie de pagos laborales. Finalmente, los administradores que desempeñan la dirección y gestión de los recursos en una empresa reciben a cambio una remuneración y la posibilidad de disfrutar unas condiciones establecidas de manera particular por la naturaleza de su cargo. 
De acuerdo con la naturaleza y actividad económica de las organizaciones, se pueden determinar relaciones con otros agentes, tales como los jubilados o pensionados, la comunidad social, los medios de comunicación, la sociedad civil, entre otros.

A partir de lo anterior, la formulación de los contratos que sustentan las relaciones entre la empresa y los diferentes grupos de interés involucran una serie de derechos y contribuciones entre ambas partes, los cuales se determinan de acuerdo a los intereses de unos y otros, como se muestra en la siguiente tabla:

Tabla 1. Contribuciones y derechos de los agentes que contratan con la empresa

\begin{tabular}{|l|l|l|}
\hline \multicolumn{1}{|c|}{ Tipo de agente } & \multicolumn{1}{c|}{ Contribución } & \multicolumn{1}{c|}{ Derechos } \\
\hline Accionista & Capital accionario & Dividendo, valor residual \\
\hline Administrador & Habilidades & Salario, bonificaciones, prestaciones \\
\hline Empleado & Habilidades & Salario, sueldos, prestaciones \\
\hline Vendedor & Bienes o servicios & Efectivo \\
\hline Cliente & Efectivo & Bienes o servicios \\
\hline Prestamista o acreedor & Capital en préstamo & Intereses, capital principal \\
\hline Gobierno & Bienes públicos & Impuestos \\
\hline Auditor & Servicios & Honorarios \\
\hline
\end{tabular}

Fuente: Sunder, S. (2005). Teoría de la contabilidad y el control. Bogotá: Universidad Nacional de Colombia, Facultad de Ciencias Económicas, Doctorado en Ciencias Económicas.

La totalidad de estas relaciones contractuales expuestas, así como aquellas otras que surjan, pueden no estar presentes en todas las organizaciones. De igual forma, tales relaciones no tendrán el mismo grado de importancia de una empresa a otra. Esto último ha sido estudiado desde la teoría de los Stakeholders ${ }^{2}$, en la cual la relación e importancia de los grupos de interés en una organización pueden no ser las mismas.

Toca (2007), a partir de estudios desarrollados por Brugha y Varvasovszky (2000), Pesqueux y Damak (2004), Carroll (2000) y Lozano (2004), propone una clasificación de Stakeholders que permite tipificar, a partir de una serie de criterios, a los distintos agentes que interactúan con las organizaciones, con lo cual se puede determinar la importancia de cada uno de ellos, como se muestra en la siguiente tabla.

2 La teoría de los Stakeholders de Freeman (1984) plantea que la empresa se relaciona con un importante número de agentes en sus actividades normales, desarrollando vínculos que pueden afectarla o beneficiarla. 
Tabla 2. Clasificación de los Stakeholders

\begin{tabular}{|c|c|c|}
\hline Criterio & $\begin{array}{c}\text { Tipos de } \\
\text { Stakeholders }\end{array}$ & Descripción \\
\hline \multirow{2}{*}{ Riesgo } & Voluntarios & $\begin{array}{l}\text { Ostentan un riesgo por tener invertido algo } \\
\text { de valor en la organización. }\end{array}$ \\
\hline & Involuntarios & $\begin{array}{l}\text { Expuestos al riesgo como resultado de las } \\
\text { actividades de la organización. }\end{array}$ \\
\hline \multirow[b]{2}{*}{ Importancia } & $\begin{array}{l}\text { Primarios o } \\
\text { contractuales }\end{array}$ & $\begin{array}{l}\text { Esenciales para la supervivencia y bienestar } \\
\text { de la organización; mantienen una relación } \\
\text { formal, oficial directa y contractual; ejercen } \\
\text { una autoridad reguladora u otras formas de } \\
\text { poder sobre la organización. }\end{array}$ \\
\hline & $\begin{array}{l}\text { Secundarios o } \\
\text { difusos }\end{array}$ & $\begin{array}{l}\text { Actores con los que interactúa la organi- } \\
\text { zación, pero que no son esenciales para su } \\
\text { supervivencia, ubicados en las fronteras de } \\
\text { la organización; pueden ser afectados por } \\
\text { sus acciones sin tener ninguna conexión } \\
\text { contractual. }\end{array}$ \\
\hline \multirow{3}{*}{ Ubicación } & Internos & $\begin{array}{l}\text { Operan dentro de los límites de la organi- } \\
\text { zación, están vinculados a ella. }\end{array}$ \\
\hline & Interfase & $\begin{array}{l}\text { Encargados de interactuar con el ambiente } \\
\text { externo. }\end{array}$ \\
\hline & Externos & $\begin{array}{l}\text { Ajenos a la organización, pero interesados } \\
\text { en su funcionamiento y en contribuir a la } \\
\text { competitividad de la misma. }\end{array}$ \\
\hline
\end{tabular}




\begin{tabular}{|l|l|l|}
\hline \multirow{1}{*}{ Criterio } & \multicolumn{1}{|c|}{$\begin{array}{c}\text { Tipos de } \\
\text { Stakeholders }\end{array}$} & \multicolumn{1}{c|}{ Descripción } \\
\hline \multirow{5}{*}{ Fuerza } & Institucionales & $\begin{array}{l}\text { Relacionados con leyes, regulaciones, enti- } \\
\text { dades inter-organizacionales y asociaciones } \\
\text { profesionales, que pueden ser específicos } \\
\text { para una industria determinada. }\end{array}$ \\
\cline { 2 - 3 } & Económicos & $\begin{array}{l}\text { Operan en los mercados de la organización } \\
\text { en cuestión. }\end{array}$ \\
\cline { 2 - 4 } & Éticos & $\begin{array}{l}\text { Emanan de grupos de presión políticos y } \\
\text { moralistas. }\end{array}$ \\
\hline \multirow{2}{*}{$\begin{array}{l}\text { Intereses } \\
\text { morales y } \\
\text { económicos }\end{array}$} & Societales & $\begin{array}{l}\text { Preocupaciones centradas en la sociedad } \\
\text { en general. }\end{array}$ \\
\cline { 2 - 4 } & De negocios & Tienen algo en juego en la organización. \\
\hline \multirow{5}{*}{ Dimensión } & Esenciales & $\begin{array}{l}\text { Subconjunto específico fundamental para la } \\
\text { supervivencia de la organización. }\end{array}$ \\
\cline { 2 - 3 } & Estratégicos & $\begin{array}{l}\text { Constituyen un conjunto particular de } \\
\text { amenazas y oportunidades en un momento } \\
\text { determinado. }\end{array}$ \\
\cline { 2 - 3 } & Ambientales & $\begin{array}{l}\text { Otros que conforman el antecedente o am- } \\
\text { biente general de la organización. }\end{array}$ \\
\cline { 2 - 3 } &
\end{tabular}

Fuente: Toca, C. (2007). Desarrollos teóricos para la comprensión y el análisis de Stakeholders. Documentos de Administración. Bogotá: Pontificia Universidad Javeriana.

Esta clasificación permite identificar, a partir de una serie de criterios, aquellos agentes que gozan de una mayor relevancia en una organización y, por lo tanto, las relaciones que se establezcan con ellos deben ser objeto de un diseño contractual y seguimiento que permita cumplir con los fines pactados con las organizaciones.

De allí, tal como se indicó anteriormente, que la contabilidad como sistema de información deba reconocer aquellos agentes que se convierten en usuarios principales 
de la información, para efectos de reconocer el tipo de mensaje que debe prepararse y presentarse para poder diseñar y ejecutar los contratos que se establezcan, es decir, una vez identificados los usuarios principales, se continúa con el tercer paso que propone Hendriksen (1981), consistente en identificar el tipo de información que debe generarse desde la contabilidad.

Al respecto, se ha planteado que de acuerdo con el mensaje que se emita desde la contabilidad y con la forma como éste se representa, surgen distintos modelos de contabilidad, los cuales individualmente o de manera conjunta pueden satisfacer las distintas necesidades de información de los grupos de interés en las organizaciones.

\section{Tipos de modelos contables}

En palabras de Gómez, el modelo contable se puede entender desde la contabilidad financiera como

la combinación particular de criterios que configuran un patrón de reconocimiento, medición y revelación de la información contable [...] Estos lineamientos definen las normas contables prescribiendo los criterios específicos de reconocimiento (pautas de registro, clasificación y categorías como noción de activo, p. ej.), medición (criterios de medida y valor, con o sin ajustes por inflación, valores de entrada o valores de salida) y revelación (especificidades de política contable, criterios de amortización, depreciación y extinción, tasación de diferencias por opciones de criterios alternativos, entre otros).

Así, un modelo contable financiero es una configuración pragmática de criterios que "prescriben" los parámetros del proceso contable, para representar la "realidad" económica y financiera de un ente (2007: 95).

La definición de los criterios enunciados anteriormente están en función de la estructura contextual, sustentada en el entorno que configura las necesidades y objetivos de los usuarios, como también en los propósitos, las restricciones del sistema contable y las condiciones estructurales de la contabilidad, lo cual, en su 
conjunto, puede configurar distintos modelos contables, como por ejemplo el modelo de contabilidad financiera (del cual parte la explicación), el de contabilidad gerencial, el de contabilidad tributaria, el de contabilidad pública y el de contabilidad ambiental, entre otros.

Estos modelos, al reconocer distintos criterios, tal como lo señala Gómez, "permiten configurar la contabilidad en un entorno concreto, para satisfacer unas necesidades específicas de información, en un marco de restricciones particulares" (2007: 96).

Sunder (2005), desde la teoría contractual de la contabilidad y el control, ha descrito en términos generales tres modelos de contabilidad, a partir del tamaño y la forma de la organización o del conjunto de contratos a los cuales presta sus servicios la contabilidad, a saber: un modelo de contabilidad basado en la teneduría de libros, un modelo de contabilidad administrativa y un modelo de contabilidad basado en información financiera.

El primero de estos modelos reconoce aquellas empresas comerciales que son manejadas por su propietario, con poca o ninguna ayuda externa, en la cual, por sus operaciones, los agentes que se identifican son el dueño, el recaudador de impuestos, los clientes, los proveedores y, en algunos casos, los acreedores financieros. El tipo de contabilidad que se adecúa a este tipo de empresas es aquella que se basa en el registro para llevar la memoria de las operaciones y la conveniente organización de los datos. Desde este modelo, algunos agentes a los cuales les interesa la información de la empresa son el recaudador de impuestos y los acreedores financieros, para efectos de determinar cargas tributarias y la capacidad de pago para afrontar obligaciones.

El segundo modelo reconoce una empresa que ha crecido y que no puede ser administrada y operada solamente por el dueño, pero en la que el interés residual es todavía cerrado, es decir, la participación de otras personas como propietarios no es una evidencia común en este tipo de organizaciones. De allí que si bien se sigue utilizando la teneduría de libros para tener memoria de las operaciones, se incluyen también otros elementos como son los presupuestos y las asignaciones interdepartamentales e intertemporales de costos y beneficios, entre otros, que permiten evaluar y controlar el desempeño de los agentes que intervienen en la administración y operación de la empresa. 
El tercer y último modelo supone una empresa internamente descentralizada, en la cual el número de accionistas ha crecido de forma tal que estos ya no pueden ejercer un control directo sobre las actividades de los administradores, por lo cual la contabilidad reconoce los dos anteriores modelos y agrega un elemento importante en este escenario: la información auditada, la cual deberá ser verificada por un tercero.

De esta manera, un modelo de contabilidad responde a unas formas de organización y necesidades de información concretas, pero se debe reconocer que en algunos casos el satisfacer dichas necesidades requiere del concurso de varios de los modelos existentes, sin que se dé una condición de exclusión entre ellos.

\section{Antecedentes de investigación}

A pesar de lo relativamente reciente que parezca ser el tema de contabilidad simplificada en Colombia y a nivel mundial, existen diversos trabajos que abordan está temática desde diferentes puntos de vista. A nivel internacional se pueden mencionar algunos desarrollos, como, por ejemplo, el de Sanz (2005), que explora las implicaciones que tiene para la empresa la utilización de la contabilidad simplificada, y el de Lima (2007), que analiza, entre otras cosas, el papel de este tipo de contabilidad para efectos de la generación de información financiera en las Pymes, en tanto herramienta para mejorar la organización de las pequeñas y medianas empresas y de las decisiones que éstas toman.

Otro de los trabajos que resulta importante mencionar es el desarrollado por Millán (2007), documento en el cual se describen los principales cambios encontrados en la reforma contable que se hizo en España en el año 2007 y, en particular, diferentes aspectos de interés relacionados con la denominada "Contabilidad Simplificada y Abreviada”, como, por ejemplo, las circunstancias establecidas para autorizar la presentación de cuentas anuales abreviadas.

Trabajos como los de Serrano (2004) exploran las novedades más importantes en cuanto a contabilidad simplificada, en lo que se refiere a las cuentas, registros, estados financieros y, principalmente, las aportaciones e inconvenientes que dicho régimen presenta para las empresas. De otra parte, el trabajo de Constans (2007) 
aborda la contabilidad simplificada en España desde la óptica de las Normas Internacionales de Información Financiera para Pymes. Finalmente, Martínez et ál. (2004) estudian el impacto práctico que tiene la contabilidad simplificada sobre las empresas y analizan la disminución de obligaciones contables que para las entidades de pequeña dimensión tiene dicho régimen.

En Colombia no existen muchos trabajos al respecto, pero se puede citar la investigación de Ruiz (2009), estudio en el cual se examinan las necesidades contables y de gestión que tienen las MiPymes en Bogotá. Aunque este trabajo no aborda directamente el tema de la contabilidad simplificada, sus resultados son importantes en el contexto de lo que ésta debería ofrecer a este tipo de organizaciones y de lo que realmente estas empresas necesitan en términos de información.

Otro de los trabajos realizados en Colombia, que sigue la misma línea del estudio comentado en el párrafo anterior, es el de Rueda et ál. (2008), documento en el cual se analiza la información contable que requieren las Pymes nacionales en el entorno de competitividad global actual. Específicamente este trabajo presenta una propuesta de un sistema de información contable integrado que cubra las necesidades que en cuanto a información contable tienen las pequeñas y medianas empresas del país. Al igual que en el trabajo anterior, dicho modelo cobra especial relevancia en el ámbito de lo que se espera de la contabilidad simplificada y lo que ésta ofrece a las empresas.

Finalmente podemos citar a nivel nacional el trabajo de Franco (1999), en el cual se aborda el estudio de la contabilidad simplificada para el sector campesino y el de la economía informal. En este documento se presenta un manual de contabilidad simplificada dirigido a este tipo de comerciantes. Esta guía fue presentada al Departamento Administrativo Nacional de Cooperativas (DANCOOP), quien lo encontró aplicable y ajustado a las normas emanadas por dicha entidad para todas aquellas unidades cooperativas interesadas en su aplicación.

Como complemento a lo anterior, en la siguiente tabla se detallan los principales trabajos relacionados con contabilidad simplificada tanto a nivel internacional como en Colombia. 
Tabla 3. Resumen de investigaciones previas sobre contabilidad simplificada

\begin{tabular}{|c|c|c|}
\hline Año & Autores & Título \\
\hline 2009 & Rosmira Ruiz García & $\begin{array}{l}\text { Estudio de las necesidades } \\
\text { contables y gestión que pre- } \\
\text { sentan las MiPymes ubicadas } \\
\text { en la localidad de Santafé de } \\
\text { Bogotá, D.C. }\end{array}$ \\
\hline 2008 & $\begin{array}{l}\text { Gabriel Rueda Delgado } \\
\text { Braulio Adriano Rodríguez Castro } \\
\text { Martha Liliana Arias Bello } \\
\text { Nancy Andrea Giraldo Ávila } \\
\text { Aracely Sánchez Serna } \\
\text { Fabiola Torres Agudelo }\end{array}$ & $\begin{array}{l}\text { Información contable integra- } \\
\text { da para la competitividad glo- } \\
\text { bal de PYMES nacionales. Una } \\
\text { aproximación conceptual. }\end{array}$ \\
\hline 2007 & Adolfo Millán Aguilar & $\begin{array}{l}\text { Principales cambios en la } \\
\text { reforma contable. }\end{array}$ \\
\hline 2007 & Anselm Constans & $\begin{array}{l}\text { La NIIF para PYMES y la Con- } \\
\text { tabilidad Simplificada. }\end{array}$ \\
\hline 2006 & Rafael Lima Fosado & $\begin{array}{l}\text { Información financiera en las } \\
\text { PYMES. }\end{array}$ \\
\hline 2006 & $\begin{array}{l}\text { Elena Merino Madrid } \\
\text { Jesús Antonio Sánchez Araque } \\
\text { Susana Villaluenga de Gracia }\end{array}$ & $\begin{array}{l}\text { La aplicación real de la Ley } \\
\text { de Nueva Empresa y la Con- } \\
\text { tabilidad Simplificada para } \\
\text { las PYME. }\end{array}$ \\
\hline 2005 & Carlos Javier Sanz Santaloria & $\begin{array}{l}\text { Contabilidad Simplificada: } \\
\text { análisis e implicaciones en la } \\
\text { empresa. }\end{array}$ \\
\hline
\end{tabular}




\begin{tabular}{|c|c|c|}
\hline Año & Autores & Título \\
\hline 2004 & José Miguel Embid Irujo & $\begin{array}{l}\text { Aproximación al régimen ju- } \\
\text { rídico de la Sociedad Limitada } \\
\text { Nueva Empresa. }\end{array}$ \\
\hline 2004 & Ángel Marina García-Tuñón & $\begin{array}{l}\text { Información y contabilidad: } \\
\text { una reflexión sobre su reciente } \\
\text { regulación legal. }\end{array}$ \\
\hline 2004 & Francisco Serrano Moracho & $\begin{array}{l}\text { Contabilidad Simplificada: } \\
\text { ¿avance o retroceso? Caracte- } \\
\text { rísticas de la Sociedad Limita- } \\
\text { da Nueva Empresa. }\end{array}$ \\
\hline 2004 & $\begin{array}{l}\text { Antonio Manuel Martínez Arias } \\
\text { Elena Fernández Rodríguez } \\
\text { José Antonio Garay González }\end{array}$ & $\begin{array}{l}\text { La Contabilidad Simplificada: } \\
\text { el impacto práctico en las } \\
\text { empresas. }\end{array}$ \\
\hline 2004 & $\begin{array}{l}\text { Ángel Alonso Pérez } \\
\text { Raquel Pousa Soto }\end{array}$ & $\begin{array}{l}\text { Contabilidad Simplificada y } \\
\text { Nueva Empresa. }\end{array}$ \\
\hline 2004 & $\begin{array}{l}\text { Inmaculada Alonso Carrillo } \\
\text { María Ángela Jiménez Montañés }\end{array}$ & $\begin{array}{l}\text { La Contabilidad Simplificada: } \\
\text { un avance a la globalización } \\
\text { en contra de la Metodología } \\
\text { Contable. }\end{array}$ \\
\hline 2004 & $\begin{array}{l}\text { Raquel Pousa Soto } \\
\text { Ángel Alonso Pérez }\end{array}$ & $\begin{array}{l}\text { Contabilidad Simplificada: un } \\
\text { caso práctico. }\end{array}$ \\
\hline 1999 & Javier Franco Hernández & $\begin{array}{l}\text { Contabilidad Simplificada } \\
\text { para formas asociativas, mi- } \\
\text { croempresas y negocios. }\end{array}$ \\
\hline
\end{tabular}

Fuente: elaboración propia 


\section{Definición y características de la contabilidad simplificada}

A partir de los anteriores aspectos es necesario indagar sobre lo definido como contabilidad simplificada, así como entender sus características de acuerdo con las aplicaciones que dicho concepto ha tenido en países como Colombia y España.

Resulta fundamental indicar que al menos para el caso de Colombia dicho concepto es eminentemente tributario, como bien es indicado en el Decreto 422 de 1991, norma en la cual se reglamenta parcialmente el Estatuto Tributario. Específicamente en el artículo primero de esta normativa se especifica lo que se entiende por contabilidad simplificada para efectos tributarios: "La Contabilidad Simplificada consiste en conservar las facturas de compra de bienes y servicios y llevar el libro fiscal de registro de operaciones diarias, en el que se consignen los ingresos diarios de cada establecimiento de comercio".

De hecho, en Colombia la contabilidad simplificada más que un modelo es un régimen tributario que brinda algunas "ventajas y facilidades" a aquellos contribuyentes que a él se acojan, previo cumplimiento de una serie de requisitos que se estipulan en el artículo segundo del decreto anteriormente comentado. Dentro de las facilidades se pueden citar, como ejemplo, la no obligatoriedad de expedir facturas por las operaciones realizadas y la no obligatoriedad de inscribir el libro de registro de operaciones diarias ante ningún ente de vigilancia y control del Estado.

Resulta importante destacar que estas supuestas ventajas y facilidades podrían llegar a contribuir aún más en el crecimiento de la economía subterránea y la inadecuada formalización de los pequeños empresarios, optando por el facilismo y la comodidad en la marcha de sus negocios y no por la adecuada formación y educación contable y financiera que permita un correcto desempeño y desarrollo por parte de las organizaciones colombianas.

Un punto de vista distinto al tributario acerca de lo que es la contabilidad simplificada es el expresado por el Consejo Técnico de la Contaduría Pública (CTCP), entidad que en su Concepto No. 13 del 16 de junio de 2009 establece que la contabilidad simplificada es el conjunto de mejores prácticas contables internacionales que se 
aplican a las micro, pequeñas y medianas empresas (MiPymes) que no cotizan en bolsa (no son entidades públicas desde la perspectiva de la conformación de capital y de su vinculación a un mercado activo de valores).

Según el CTCP, la contabilidad simplificada supone una serie de directrices y lineamientos caracterizados, principalmente, por un menor requerimiento de información a emitir, de tal forma que no se generen exageradas obligaciones para las micro, pequeñas y medianas empresas, que entorpezcan su normal desarrollo.

De otra parte, si se explora el concepto de contabilidad simplificada en España, se tiene que observar lo establecido en el Real Decreto 296/2004 del 20 de febrero, en el cual se aprueba el régimen simplificado de contabilidad. Este decreto tiene su origen en la Ley Séptima de 2003 del 1 de abril, en la cual se establece la aprobación reglamentaria para nuevas formas jurídico-societarias, que tiene como principal objetivo la aplicación del principio de simplificación.

De acuerdo con lo anterior, puede deducirse que la contabilidad simplificada en España, al igual que en Colombia, está dirigida para pequeños empresarios que no superan ciertos niveles de ingresos y activos (entre otros requisitos), de tal manera que estas compañías puedan desarrollar sus diferentes actividades con la menor cantidad posible de "obstáculos" de tipo legal, fiscal y administrativo. Al igual que para el caso Colombiano, los empresarios españoles podrán disfrutar de una serie de facilidades, como, por ejemplo, la utilización de un libro diario simplificado donde se registran los diferentes movimientos y la generación de un balance de la cuenta de pérdidas, ganancias y memoria, según los modelos simplificados establecidos en la normativa.

Es pertinente comentar que aunque el concepto de contabilidad simplificada es aplicable a pequeños empresarios, hay que tener la suficiente claridad respecto al concepto de "pequeño empresario", tanto en Colombia como en España. De hecho, ambas normativas establecen una serie de características específicas que deben tener todos aquellos que quieran pertenecer a este régimen.

Retomando la norma tributaria colombiana (Decreto 422 de 1991), se establece que sólo podrán aplicar contabilidad simplificada aquellos contribuyentes que no estén 
constituidos como sociedad, que sus ingresos netos en el año fiscal inmediatamente anterior no superen la suma de $\$ 12.200 .000$ (año base 1990), que su patrimonio bruto fiscal a 31 de diciembre del año inmediatamente anterior no sea superior a \$33.900.000 (año base 1990), que no tengan más de dos establecimientos de comercio y, finalmente, que en el caso de los responsables del impuesto sobre las ventas, no estén inscritos en el régimen común.

En cuanto a la norma española (Real Decreto 296/2004), se establece como requisito para pertenecer a este régimen el cumplimiento de al menos dos de las siguientes circunstancias:

- Que el total de las partidas del activo en el que han de incluirse los compromisos financieros pendientes no superen un millón de euros para empresarios o ciento cincuenta mil en el caso de fundaciones y asociaciones.

- Que el importe neto de su cifra anual de negocios sea inferior a dos millones de euros en empresarios o a ciento cincuenta mil en el caso de fundaciones y asociaciones.

- Que el número medio de trabajadores empleados en el ejercicio no sea superior a 10 en el supuesto de empresarios o a 5 en el resto de entidades.

Si se comparan los requisitos de ingresos según la norma colombiana, estos se encuentran por debajo del importe neto de la cifra anual de negocios establecida en la normativa española, por lo cual lo que se entiende por pequeños empresarios o comerciantes varía sensiblemente de un país a otro.

Aunque si por el contrario establecemos esta comparación por el número de empleados, las cifras son básicamente las mismas, teniendo en cuenta que respecto al número de trabajadores, para el caso de España se toma el criterio estipulado por la Unión Europea para sus países miembro, como se muestra en la siguiente tabla. 
Tabla 4. Clasificación de empresas según el número de empleados

\begin{tabular}{|l|l|l|}
\hline \multicolumn{1}{|c|}{ Rango } & \multicolumn{1}{|c|}{ Normativa colombiana } & \multicolumn{1}{c|}{ Normativa europea } \\
\hline Microempresas & Máximo 10 empleados & Entre 1 y 9 empleados \\
\hline Pequeñas empresas & Entre 11 y 50 empleados & Entre 10 y 49 empleados \\
\hline Medianas empresas & Entre 51 y 200 empleados & Entre 50 y 249 empleados \\
\hline Grandes empresas & Más de 200 empleados & $\begin{array}{l}\text { De } 250 \text { empleados en } \\
\text { adelante }\end{array}$ \\
\hline
\end{tabular}

Fuente: elaboración propia a partir de la Ley 905 de 2004 (Colombia) y de la normativa europea

A partir de lo estudiado, es posible concluir que la contabilidad simplificada no puede abordarse y entenderse simplemente desde el punto de vista fiscal, es decir, como un régimen tributario especial destinado a determinadas compañías que cumplan una serie de requisitos y características específicas, sino que debe comprenderse como un sistema de información contable que busca facilitarle a las MiPymes su funcionamiento, desarrollo y cumplimiento de los objetivos. Sin embargo, vale aclarar, como se comentaba en párrafos precedentes, que dicho sistema puede ser puerta de entrada a diversos problemas, tales como la economía subterránea y la no adecuada formalización de los pequeños empresarios.

Por otra parte, resulta importante mencionar que organismos como el Consejo de Normas Internacionales de Contabilidad (IASB) y la Conferencia de las Naciones Unidas sobre Comercio y Desarrollo (UNTACD) ${ }^{3}$ plantean unos cuerpos normativos que emiten una serie de lineamientos para la preparación y presentación de la información contable y financiera en empresas con características propias de las Pymes, que, en últimas, son las que utilizarían un sistema de contabilidad simplificada.

3 Es importante aclarar que el IASB y la UNTACD no han declarado específicamente que la normatividad emitida corresponda a un sistema específicamente denominado "contabilidad simplificada". 
Para el caso del IASB, este organismo desarrolló y publicó las Normas Internacionales de Información Financiera para pequeñas y medianas empresas (Pymes). Estas normas se aplican sobre los estados financieros, con propósito de manejar información general y otros tipos de información contable. El IASB entiende por pequeñas y medianas empresas todas aquellas organizaciones que no tienen la obligación pública de rendir cuentas y que la publicación de sus estados financieros es realizada con propósitos de información general para usuarios externos.

En cuanto al modelo de ISAR (UNTACD), se reconoce la existencia de tres tipos o categorías de Pymes, los cuales lógicamente demandarán la necesidad de sistemas de contabilidad e información diferentes a los de las grandes empresas e, incluso, con distinciones entre cada uno de ellos. Estas categorías se denominan Pymes de nivel I, Pymes de nivel II y Pymes de nivel III.

En cuanto a las Pymes de nivel I -que se supone son las Pymes de mayor tamaño-, según lo detallado en UNTACD (2001), se parte del supuesto de que estas empresas deben cumplir íntegramente las IAS (Normas Internacionales de Contabilidad), por ser empresas que emiten valores en los mercados públicos.

En segunda instancia, las Pymes de nivel II están conformadas por compañías que no emiten valores públicos ni publican informes financieros destinados al público en general. A este grupo de empresas se les aplica una versión abreviada de las IAS, conformada por 15 normas:

Tabla 5. Versión abreviada de las IAS aplicadas a las Pymes de nivel II

\begin{tabular}{|l|l|}
\hline IAS & Temática abordada \\
\hline IAS 1 & Presentación de estados financieros \\
\hline IAS 2 & Inventarios \\
\hline IAS 7 & Estados del flujo de caja \\
\hline IAS 8 & $\begin{array}{l}\text { Resultado neto del ejercicio, errores fundamentales y cambios en } \\
\text { las políticas contables }\end{array}$ \\
\hline IAS 10 & $\begin{array}{l}\text { Contingencias y hechos ocurridos después de la fecha de cierre } \\
\text { del ejercicio }\end{array}$ \\
\hline
\end{tabular}




\begin{tabular}{|l|l|}
\hline IAS 12 & Impuestos sobre los beneficios \\
\hline IAS 16 & Material inmovilizado \\
\hline IAS 17 & Arrendamientos financieros \\
\hline IAS 18 & Ingresos \\
\hline IAS 20 & $\begin{array}{l}\text { Contabilización de las subvenciones públicas en información } \\
\text { sobre las ayudas públicas }\end{array}$ \\
\hline IAS 21 & $\begin{array}{l}\text { Efectos de las variaciones de las cotizaciones de las monedas } \\
\text { extranjeras }\end{array}$ \\
\hline IAS 23 & Costo de los préstamos \\
\hline IAS 24 & Información sobre las empresas vinculadas \\
\hline IAS 37 & Provisiones, pasivo contingente y activo contingente \\
\hline IAS 38 & Activo intangible \\
\hline
\end{tabular}

Fuente: elaboración propia a partir de información de UNTACD (2001)

Este grupo abreviado de normas, en palabras del Grupo Consultivo Especial de la UNTACD, debe favorecer la eficiencia en las organizaciones donde se aplicarán dichos estándares.

Finalmente, en cuanto a las Pymes de nivel III, están constituidas por las Pymes más pequeñas, empresas que según UNTACD (2001), experimentan importantes problemas en cuanto a la obtención de créditos bancarios, créditos comerciales y el acceso a servicios de contabilidad que se ajusten a sus necesidades específicas. Según el Grupo Consultivo Especial de la UNTACD, las normas de contabilidad que se apliquen a este grupo de empresas deben tener en cuenta la sencillez de sus operaciones, la falta de recursos y los conocimientos e infraestructura limitados en cuanto a contabilidad se refiere. 
En concordancia con lo anterior, el Grupo Consultivo Especial recomienda que se utilice el sistema de la contabilidad acumulativa simple, que sigue casi los mismos lineamientos de la IAS 1. Las Pymes de nivel III no estarían en la obligación de cumplir las IAS, aunque hay que reconocer que dicho sistema estaría basado en el método de la contabilidad a costo histórico y del valor devengado o principio de causación, tal como es planteado por UNTACD (2001).

\section{Análisis del concepto de contabilidad simplificada a la luz de los modelos contables existentes}

Teniendo en cuenta los distintos enfoques o puntos de vista respecto al concepto de contabilidad simplificada y los modelos contables anteriormente reseñados, resulta pertinente analizar cómo las diversas interpretaciones que se hacen acerca de la contabilidad simplificada se relacionan y responden a las características y particularidades de los diferentes modelos existentes.

Independientemente de cómo se identifique a la contabilidad simplificada, es fundamental reconocer que este sistema de contabilidad nace con un objetivo muy claro y definido: facilitarle a las empresas el desarrollo de sus actividades para un adecuado cumplimiento de sus metas organizacionales, mediante la eliminación de "trabas" administrativas, fiscales, legales, etc. que obstaculicen su desempeño.

Partiendo de ese reconocimiento inicial, puede señalarse que a la luz de los criterios que conforman la estructura de uno u otro modelo contable en particular, los cuales permiten constituir la contabilidad en un determinado ambiente y resolver una serie de necesidades específicas de información, la contabilidad simplificada pretende ese mismo objetivo, es decir, adaptarse a una serie de características especiales que tiene un determinado grupo de empresas que por sus particularidades requieren de un sistema de contabilidad que les permita dar solución a sus diferentes problemáticas de la forma más eficiente y eficaz posible.

Ahora bien, si se analiza esta relación desde los modelos de contabilidad descritos por Sunder (2005): teneduría de libros, contabilidad administrativa y contabilidad financiera, resulta interesante destacar que el primero de ellos describe las 
características generales que un sistema de contabilidad simplificada debería tener; sistema en el cual lo más relevante es el sencillo registro y adecuada organización de las operaciones que realice la empresa, teniendo en cuenta el limitado número de agentes con los cuales interactúa en el desarrollo de sus actividades.

Respecto al segundo y tercer modelo planteado por Sunder (2005), en nuestra opinión, sus características y necesidades desbordan y sobrepasan lo que la contabilidad simplificada puede ofrecer, teniendo en cuenta lo que se entiende por este concepto y las necesidades específicas planteadas en los modelos sugeridos, como bien fue explicado en los apartados precedentes de este escrito.

\section{Conclusiones}

En el presente documento se exploró y analizó el concepto de contabilidad simplificada, y para ello se tomaron las experiencias y desarrollos de dos países en particular: Colombia y España, naciones que, entre otras cosas, manejan sistemas contables similares (continental europeo), lo cual permite establecer paralelos y puntos comunes entre ambos Estados. Este análisis permitió establecer qué tipo de organizaciones son las más beneficiadas con este sistema: organizaciones que atañen a las micro, pequeñas y medianas empresas de ambos países.

Igualmente se pudo establecer que un sistema de contabilidad simplificada genera una serie de ventajas y desventajas. Para el caso de las primeras, podemos mencionar como la más importante la eliminación de una serie de obligaciones u "obstáculos" de tipo legal,fiscal y administrativo, lo cual facilita la puesta en marcha y desarrollo de una organización de esta clase. De otra parte, si nos referimos a las desventajas, es claro que este sistema podría ocasionar, como bien se indicó a lo largo del documento, la no formalización de los pequeños empresarios y podría impulsar, de cierta manera, la economía subterránea.

También resulta importante resaltar que tanto para Colombia como para España la contabilidad simplificada parece entenderse solamente desde el punto de vista de lo tributario, es decir, como un sistema o régimen tributario dirigido a cierto tipo de empresas que cumplen algunas características ya definidas, lo cual, en opinión de los autores, resulta equivocado, ya que se debe comprender como un 
sistema que pretende facilitar el cumplimiento de las metas y objetivos que tienen las organizaciones de menor tamaño.

Finalmente, el tema en mención reviste de tanta importancia y trascendencia que diferentes organismos a nivel mundial emiten una serie de normatividades dirigidas específicamente a este tipo de empresas, reconociendo su amplia presencia e importancia en la economía de todos los países globalmente. Organizaciones como el IASB y la UNTACD vienen trabajando al respecto desde hace ya varios años. La primera de ellas, mediante la emisión de las Normas Internacionales de Información Financiera para pequeñas y medianas empresas (Pymes); y la segunda, mediante la emisión de un modelo que reconoce la existencia de diferentes clases de Pymes, que, a su vez, requerirán de sistemas de contabilidad e información distintos a los de las grandes compañías.

\section{Referencias}

Brugha, R. y Varvasovszky, Z. (2000). Stakeholders Analysis: A Review. Health Policy and Planning, 15(3).

Cañibano, L. (1996). Los programas de investigación en contabilidad. Revista Contaduría, 29, 13-61.

Carroll, A. (2000). Conceptual and Consulting Aspects of Stakeholder Theory, Thinking and Management. Handbook of Organizational Consultation. Nueva York: Marcel Dekker Incorporated.

Conferencia de las Naciones Unidas sobre Comercio y Desarrollo (UNCTAD) (2001). La contabilidad de las pequeñas y medianas empresas. Informe del Grupo Consultivo Especial de Expertos en contabilidad de las pequeñas y medianas empresas, $\mathrm{N}^{\circ} 12$. Ginebra.

Congreso de la República de Colombia (2004). Ley 905, por medio de la cual se modifica la Ley 590 de 2000 sobre promoción del desarrollo de la micro, pequeña y mediana empresa colombiana y se dictan otras disposiciones. 
Congreso de la República de Colombia (2009). Ley 1314, por la cual se regulan los principios y normas de contabilidad e información financiera y de aseguramiento de información aceptados en Colombia, se señalan las autoridades competentes, el procedimiento para su expedición y se determinan las entidades responsables de vigilar su cumplimiento.

Consejo de Normas Internacionales de Contabilidad (IASB) (2009). Norma Internacional de Información Financiera para Pequeñas y Mediana Entidades (PYMES). Londres: IASCF.

Consejo Técnico de la Contaduría Pública (2009). Concepto No. 13 IFRS Pymes. Recuperado de http://www.actualicese.com.

Constans, A. (2007). La NIIF para Pymes y la Contabilidad Simplificada. Revista de Contabilidad y Dirección, 4, 35-54.

España (2003). Ley 7/2003 de 1 de abril, por la que se modifica la Ley 2/1995 de 23 de marzo de Sociedades de Responsabilidad Limitada.

España (2004). Real Decreto 296/2004 de 20 de febrero, por el que se aprueba el régimen simplificado de la contabilidad.

Franco, J.(1999). Contabilidad simplificada para formas asociativas, microempresas y negocios. Bucaramanga: Centro Empresarial Chicamocha.

Freeman, E.R. (1984). Strategic Management: a Stakeholder Approach. Pitman Publishing.

Gómez, M. (2007). Comprendiendo las relaciones entre los sistemas contables, los modelos contables y los sistemas de información contables empresariales. Revista Internacional Legis de Contabilidad y Auditoría, 32, 83-13.

Hendriksen, E. (1981). Teoría de la contabilidad. Ciudad de México: Unión Tipográfica Editorial. 
Lima, R.(2007). Información financiera en las PYMES. Revista del Centro de Investigación, 7(27), 57-75. Universidad La Salle

Lozano, J. (2004). Towards the Relational Corporation: From Managing Stakeholder Relationships to Building Stakeholder Relationships. Stakeholders in Perspective. Bradford: Emerald Group Publishing.

Martínez, A., Fernández, E. y Garay, J. (2004). Harvard Deusto. Finanzas y Contabilidad, 60, 62-72.

Millán, A. (2007). Principales cambios en la reforma contable. Revista Partida Doble, 191, 22-33.

Ministerio de Hacienda y Crédito Público (1991). Decreto 422, por medio del cual se reglamente la contabilidad simplificada en Colombia para efectos tributarios.

Pesqueux, Y.y Damak, S. (2004). Stakeholder Theory in Perspective. Stakeholders in Perspective. Bradford: Emerald Group Publishing.

Presidencia de la República de Colombia (1991). Decreto 422 de 1991, por el cual se reglamenta parcialmente el Estatuto Tributario.

Presidencia de la República de Colombia (1993a). Decreto 2649 de 1993, por el cual se reglamenta la contabilidad en general y se expiden los principios o normas de contabilidad generalmente aceptados en Colombia.

Presidencia de la República de Colombia (1993b). Decreto 2650 de 1993, por el cual se modifica el Plan Único de Cuentas para los comerciantes.

Rueda, G., Rodríguez, B., Arias, M., Giraldo, N., Sánchez, A. y Torres, F. (2008). Información contable integrada para la competitividad global de PYMES nacionales. Una aproximación conceptual. La formación del Contador Público en la generación y la gestión de la información. Medellín: Universidad de Medellín. 
Ruiz, R. (2009). Estudio de las necesidades contables y gestión que presentan las MiPymes ubicadas en la localidad de Santafé de Bogotá. Bogotá: Universidad Central.

Sanz, S. (2005). Contabilidad Simplificada: análisis e implicaciones en la empresa. Revista Acciones e Investigaciones Sociales, 20, 113-122.

Serrano, F. (2004). Contabilidad Simplificada: ¿avance o retroceso? Características de la Sociedad Limitada Nueva Empresa. Revista Estrategia Financiera, 208, 20-31.

Sunder, S. (2005). Teoría de la contabilidad y el control. Bogotá: Universidad Nacional de Colombia, Facultad de Ciencias Económicas.

Toca, C. (2007). Desarrollos teóricos para la comprensión y el análisis de Stakeholders. Documentos de Administración. Bogotá: Pontificia Universidad Javeriana.

World Bank (2003). Report on the Observance of Standards and Codes: Accounting and Auditing. Bogotá: The World Bank. 